Supporting Information

\title{
Measurement of Solvation Ability of Solvents by Porphyrin-Based
}

\section{Solvation/Desolvation Indicators}

\author{
Motonobu Sugimoto, Yusuke Kuramochi, and Akiharu Satake*
}

Graduate School of Science, Tokyo University of Science, 1-3 Kagurazaka, Shinjuku-ku, Tokyo 162-86o1, Japan. 

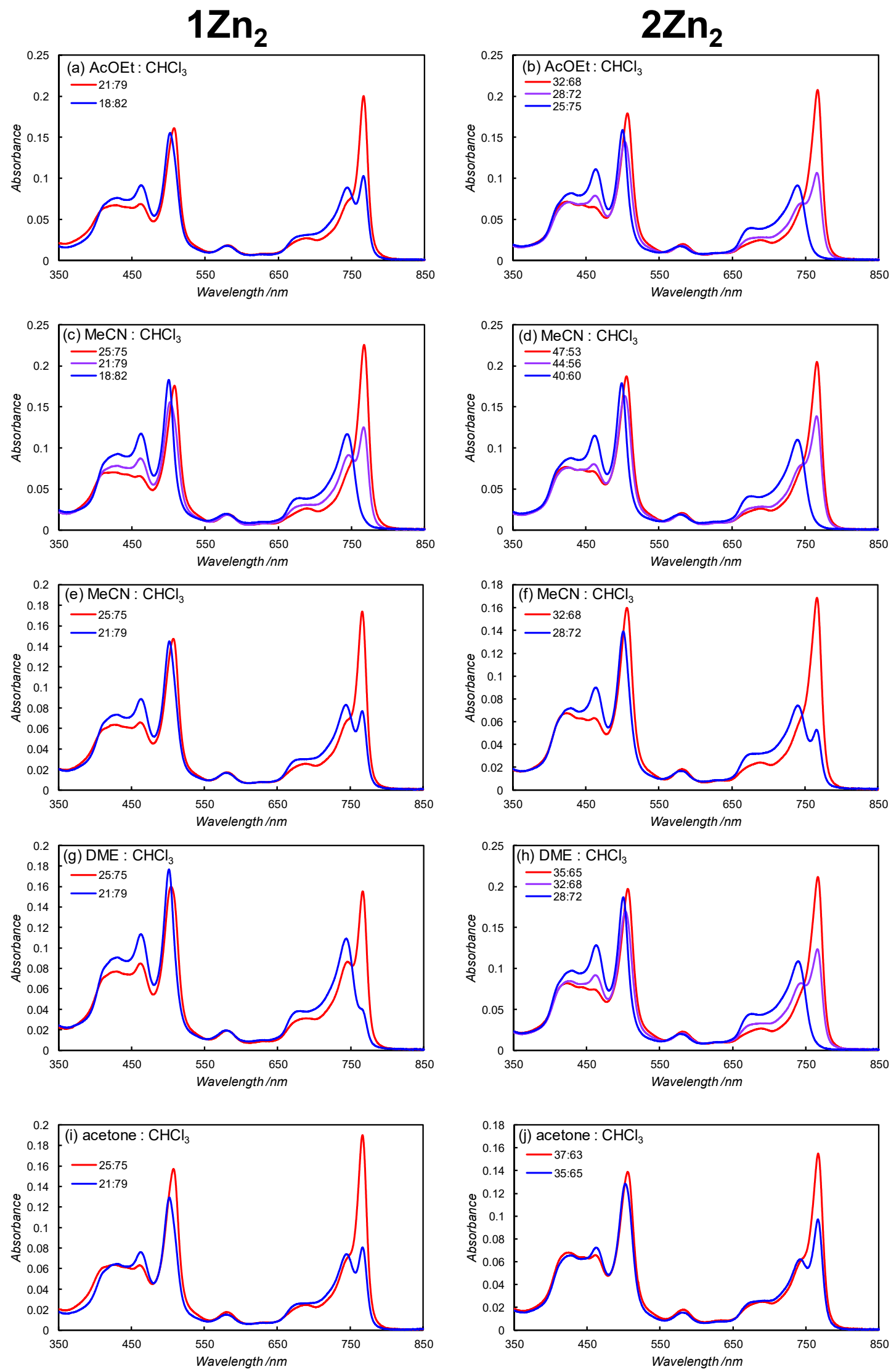

Figure S1. UV-vis spectra of $\mathbf{1} \mathbf{Z n}_{\mathbf{2}}$ (the left lane) and $\mathbf{2} \mathbf{Z n}_{\mathbf{2}}$ (the right lane) in a binary of solvent systems. (a, b) ethyl acetate and chloroform, (c, d) acetonitrile and chloroform, (e, f) $n$-butyl acetate and chloroform, (g, h) DME and chloroform, (i, j) acetone and chloroform. 

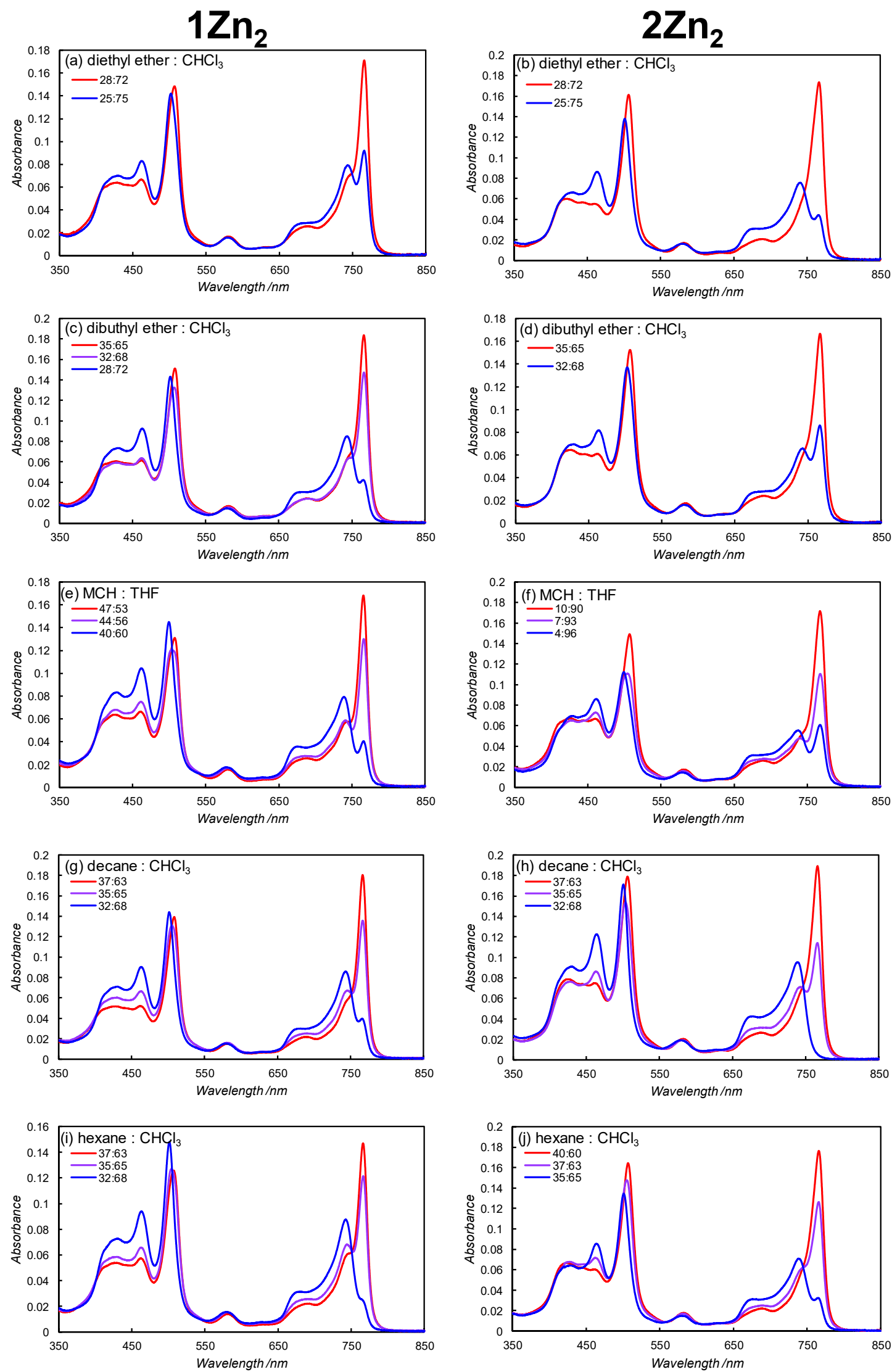

Figure S2. UV-vis spectra of $\mathbf{1} \mathbf{Z n}_{\mathbf{2}}$ (the left lane) and $\mathbf{2} \mathbf{Z n}_{\mathbf{2}}$ (the right lane) in a binary of solvent systems. (a, b) diethyl ether and chloroform, (c, d) dibutyl ether and chloroform, (e, f) MCH and THF, (g, h) $n$-decane and chloroform, (i, j) $n$-hexane and chloroform. 

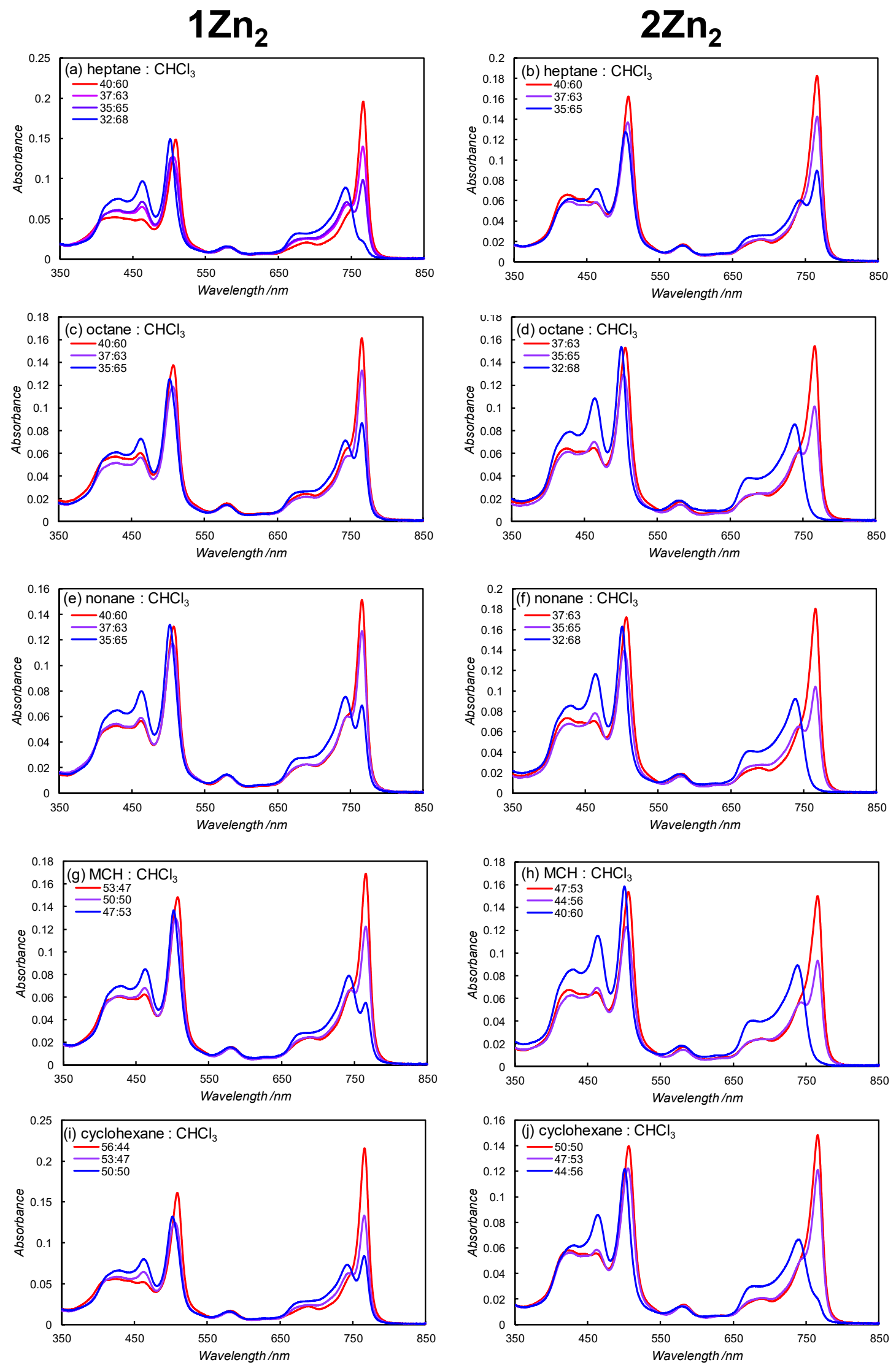

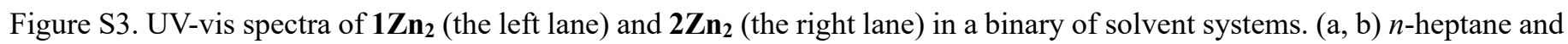
chloroform, (c, d) n-octane and chloroform, (e, f) n-nonane and chloroform, (g, h) $\mathrm{MCH}$ and chloroform, (i, j) cyclohexane and chloroform. 
$1 \mathrm{Zn}_{2}$
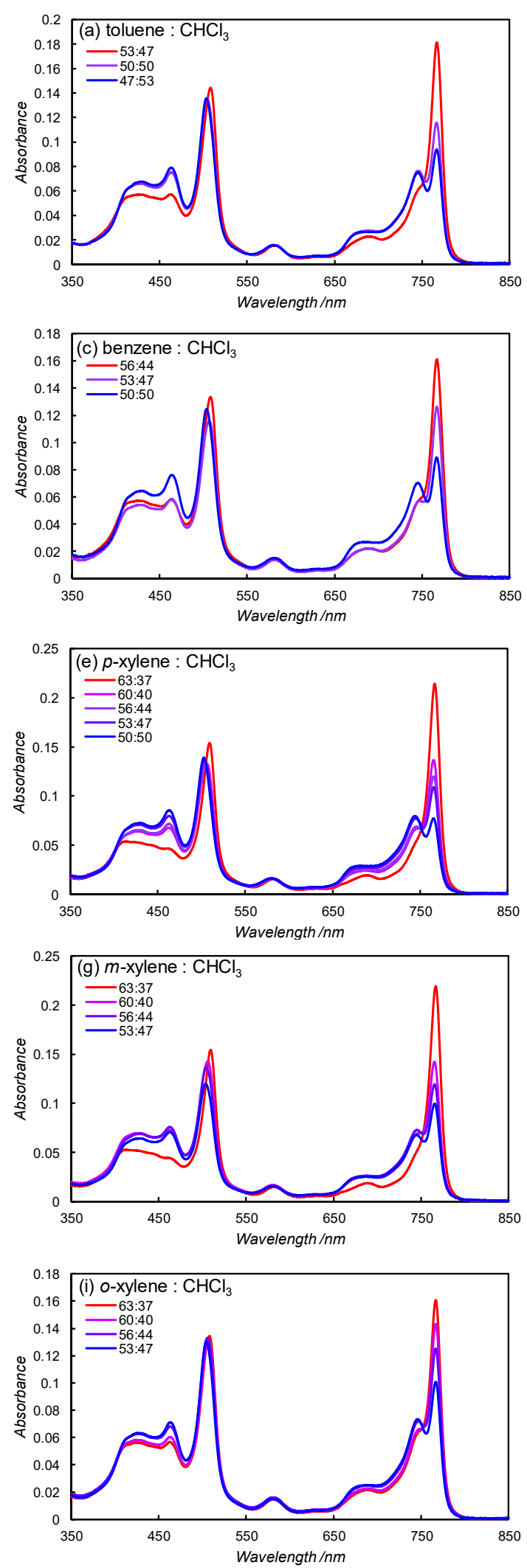

$2 \mathrm{Zn}_{2}$
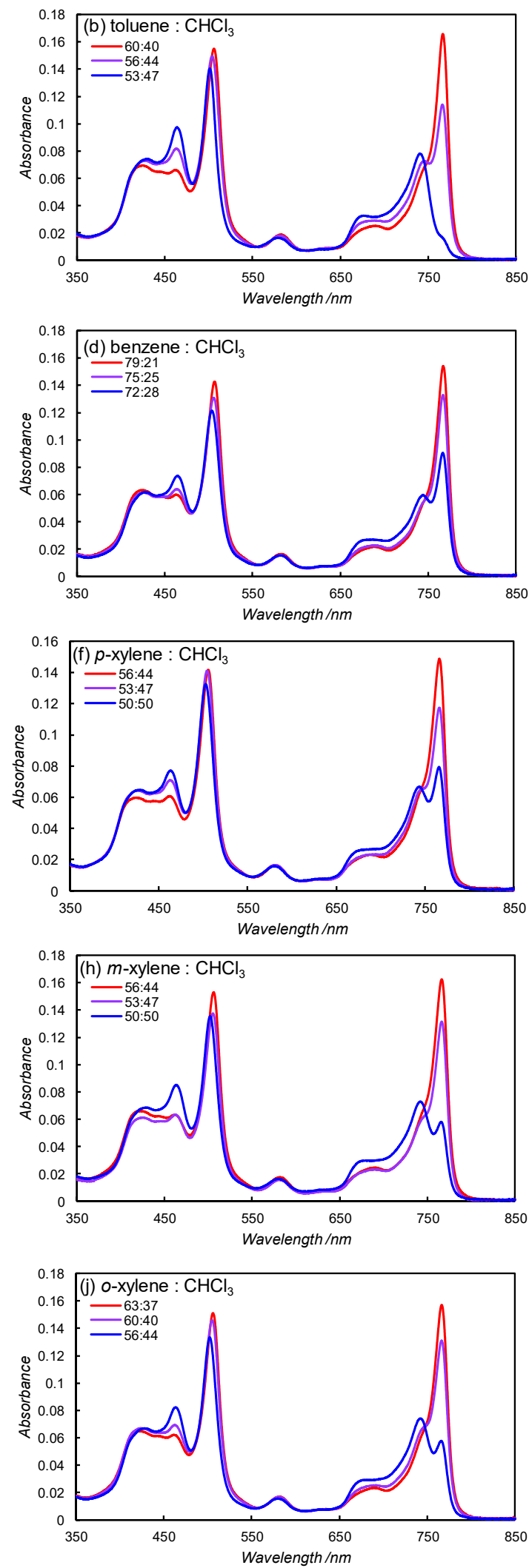

Figure S4. UV-vis spectra of $\mathbf{1} \mathbf{Z n}_{\mathbf{2}}$ (the left lane) and $\mathbf{2} \mathbf{Z n} \mathbf{n}_{\mathbf{2}}$ (the right lane) in a binary of solvent systems. (a, b) toluene and chloroform, (c, d) benzene and chloroform, (e, f) $p$-xylene and chloroform, (g, h) $m$-xylene and chloroform, (i, j) $o$-xylene and chloroform. 
$1 \mathrm{Zn}_{2}$
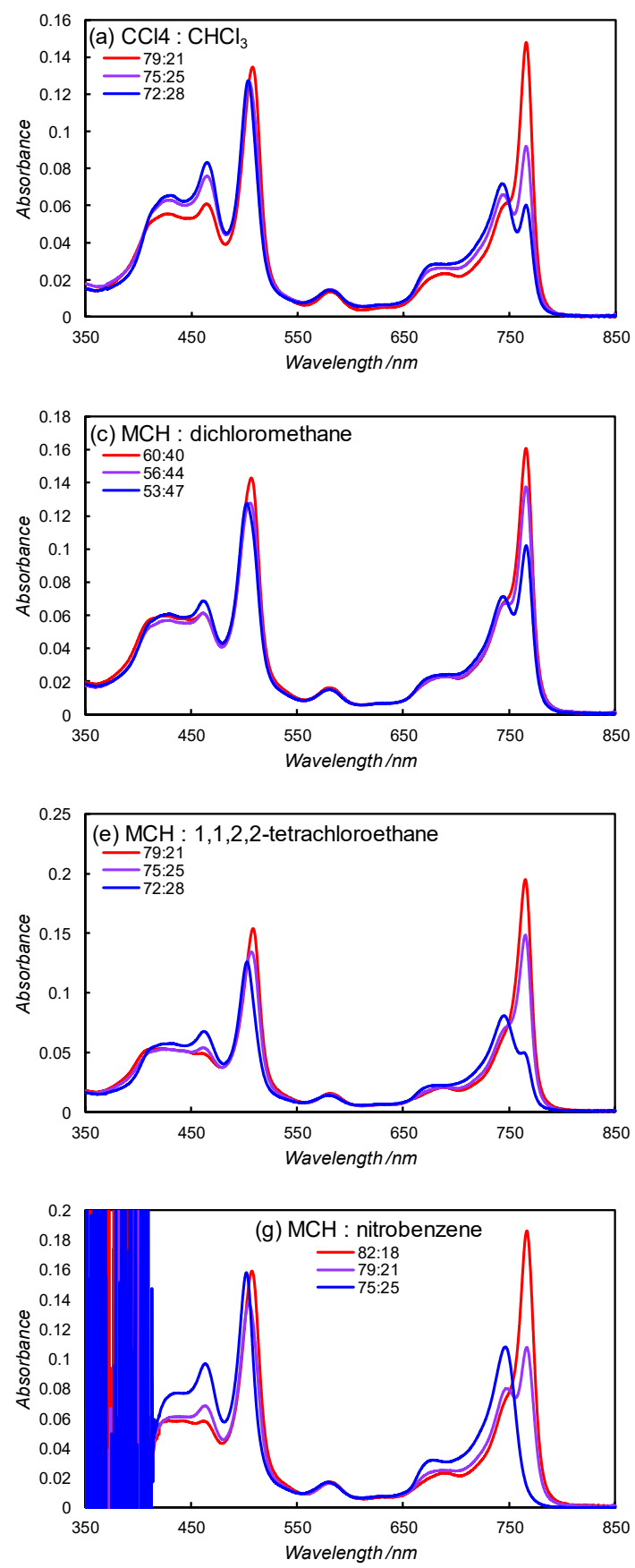

$2 \mathrm{Zn}_{2}$
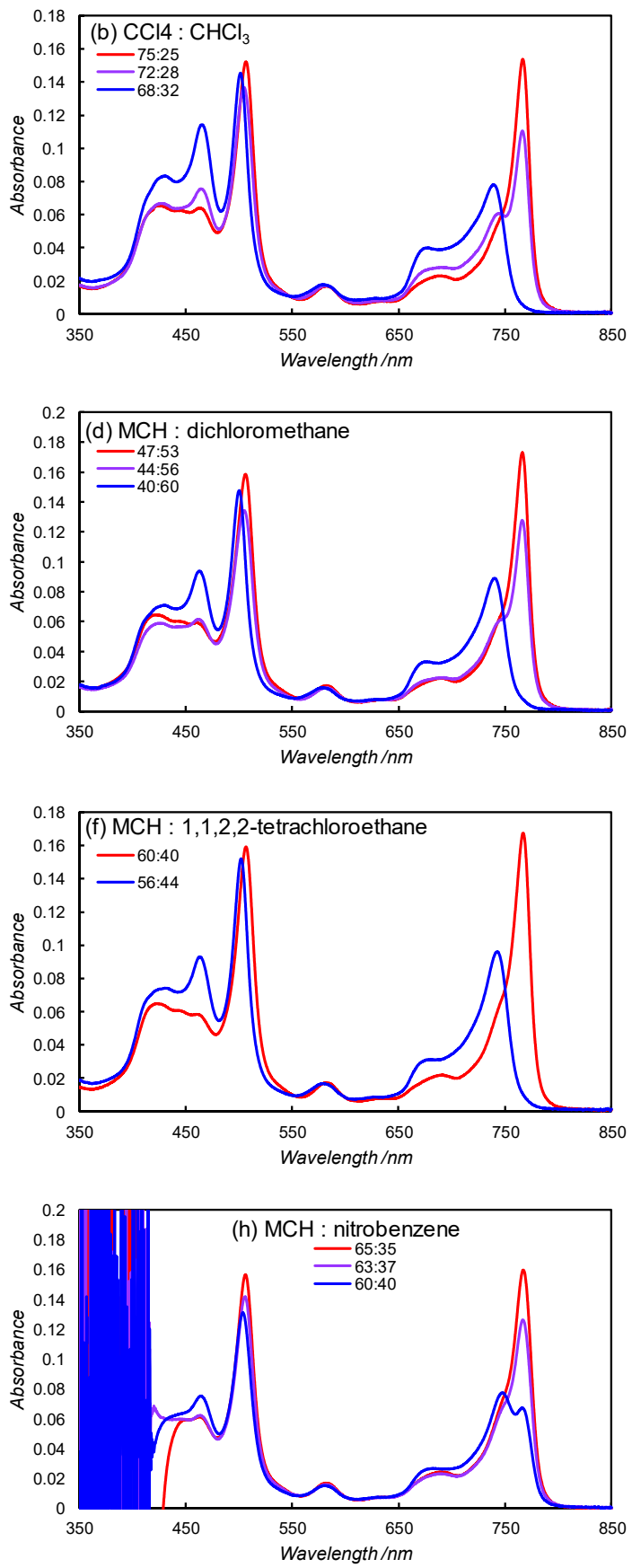

Figure S5. UV-vis spectra of $\mathbf{1} \mathbf{Z n}_{\mathbf{2}}$ (the left lane) and $\mathbf{2} \mathbf{Z n}_{\mathbf{2}}$ (the right lane) in a binary of solvent systems. (a, b) $\mathbf{C C l}_{4}$ and chloroform, (c, d) MCH and dichloromethane, (e, f) MCH and 1,1,2,2-tetrachloroethane, (g, h) MCH and nitrobenzene. 


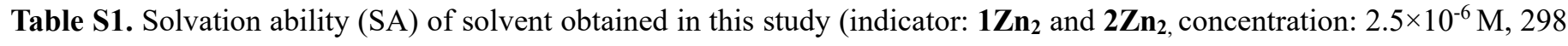
$\mathrm{K}$, Standard "good" solvent: $\mathrm{CHCl}_{3}$, standard "poor" solvent: $\left.\mathrm{MCH}\right)$. Solvent scales ${ }^{1}, \mathrm{CED}, \pi^{*}, E_{\mathrm{T}}(30)$, reflective index $(n)$, and dielectric constant $(\varepsilon)$, are also listed.

\begin{tabular}{|c|c|c|c|c|c|c|c|}
\hline & SA & SA & & & & & \\
\hline solvent & $1 \mathrm{Zn}_{2}$ & $2 Z_{2}$ & CED & $\pi^{*}$ & $E_{\mathrm{T}}(30)$ & $n$ & $\varepsilon$ \\
\hline AcOEt & $-81 \pm 2$ & $-72 \pm 4$ & 80.9 & 0.45 & 38.1 & 1.3724 & 6.03 \\
\hline $\mathrm{MeCN}$ & $-79 \pm 4$ & $-57 \pm 4$ & 140 & 0.66 & 45.6 & 1.3441 & 36 \\
\hline $\mathrm{AcOBu}$ & $-77 \pm 2$ & $-70 \pm 2$ & 74.9 & 0.46 & 38.5 & 1.395 & 5.07 \\
\hline DME & $-77 \pm 2$ & $-69 \pm 4$ & 77.4 & 0.53 & 38.2 & 1.3796 & 7.2 \\
\hline acetone & $-77 \pm 2$ & $-64 \pm 1$ & 92.8 & 0.62 & 42.2 & 1.3587 & 21.36 \\
\hline diethyl ether & $-74 \pm 2$ & $-70 \pm 2$ & 56.7 & 0.24 & 34.5 & 1.352 & 4.42 \\
\hline dibutyl ether & $-69 \pm 4$ & $-67 \pm 2$ & 60.0 & 0.24 & 33 & 1.398 & 3.18 \\
\hline THF & $44 \pm 4$ & $7 \pm 3$ & 86.3 & 0.55 & 37.4 & 1.4072 & 7.47 \\
\hline decane & $-66 \pm 3$ & $-66 \pm 3$ & 69.9 & 0.03 & 31 & 1.412 & 1.989 \\
\hline hexane & $-66 \pm 3$ & $-63 \pm 3$ & 53.1 & -0.11 & 31 & 1.375 & 1.89 \\
\hline heptane & $-64 \pm 4$ & $-63 \pm 3$ & 55.2 & -0.06 & 31.1 & 1.358 & 1.84 \\
\hline octane & $-63 \pm 3$ & $-66 \pm 3$ & 57.8 & 0.01 & 31.1 & 1.398 & 1.96 \\
\hline nonane & $-63 \pm 3$ & $-66 \pm 3$ & 58.2 & - & 31 & 1.405 & 1.97 \\
\hline $\mathrm{MCH}$ & $-50 \pm 3$ & $-57 \pm 4$ & 61.2 & 0 & 32.2 & 1.423 & 2 \\
\hline cyclohexane & $-47 \pm 3$ & $-53 \pm 3$ & 67.5 & 0 & 30.9 & 1.426 & 2.02 \\
\hline toluene & $-50 \pm 3$ & $-44 \pm 4$ & 79.2 & 0.49 & 33.9 & 1.4969 & 2.43 \\
\hline benzene & $-47 \pm 3$ & $-25 \pm 4$ & 84.5 & 0.55 & 34.3 & 1.501 & 2.4 \\
\hline$p$-xylene & $-44 \pm 7$ & $-47 \pm 3$ & 77.4 & 0.43 & 33.1 & 1.496 & 2.27 \\
\hline$m$-xylene & $-42 \pm 5$ & $-47 \pm 3$ & 77.4 & 0.47 & 33.3 & 1.497 & 2.374 \\
\hline$o$-xylene & $-42 \pm 5$ & $-41 \pm 4$ & 80.9 & 0.45 & 33.1 & 1.4969 & 2.27 \\
\hline $\mathrm{CCl}_{4}$ & $-25 \pm 4$ & $-29 \pm 4$ & 74.0 & 0.21 & 32.4 & 1.4602 & 2.3 \\
\hline chloroform & $50 \pm 3$ & $44 \pm 4$ & 85.4 & 0.69 & 39.1 & 1.4459 & 4.89 \\
\hline dichloromethane & $57 \pm 4$ & $44 \pm 4$ & 98.5 & 0.82 & 40.7 & 1.424 & 9.02 \\
\hline $\begin{array}{l}1,1,2,2- \\
\text { tetrachloroethane }\end{array}$ & $76 \pm 4$ & $58 \pm 2$ & 97.5 & 0.62 & 39.4 & 1.494 & 8.42 \\
\hline nitrobenzene & $79 \pm 4$ & $63 \pm 3$ & 122 & 0.86 & 41.2 & 1.5562 & 36.09 \\
\hline
\end{tabular}




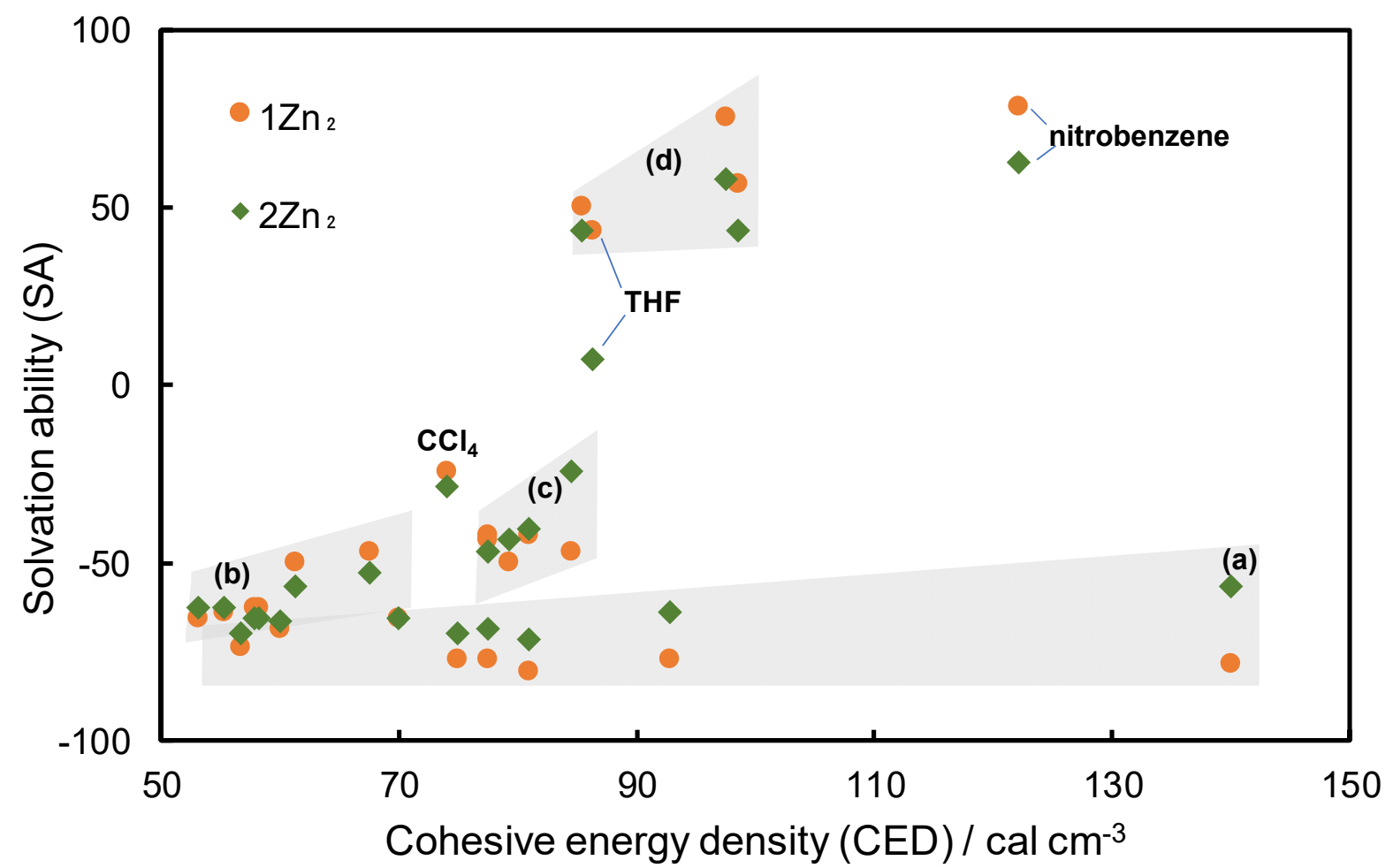

Figure S6. Relationships between SA and cohesive energy density (CED) of various solvents in Table S1. (a) polar nonaromatic compounds, (b) nonpolar and nonaromatic compounds (alkanes), (c) benzene and methylated benzenes, (d) chloro-organic compounds.

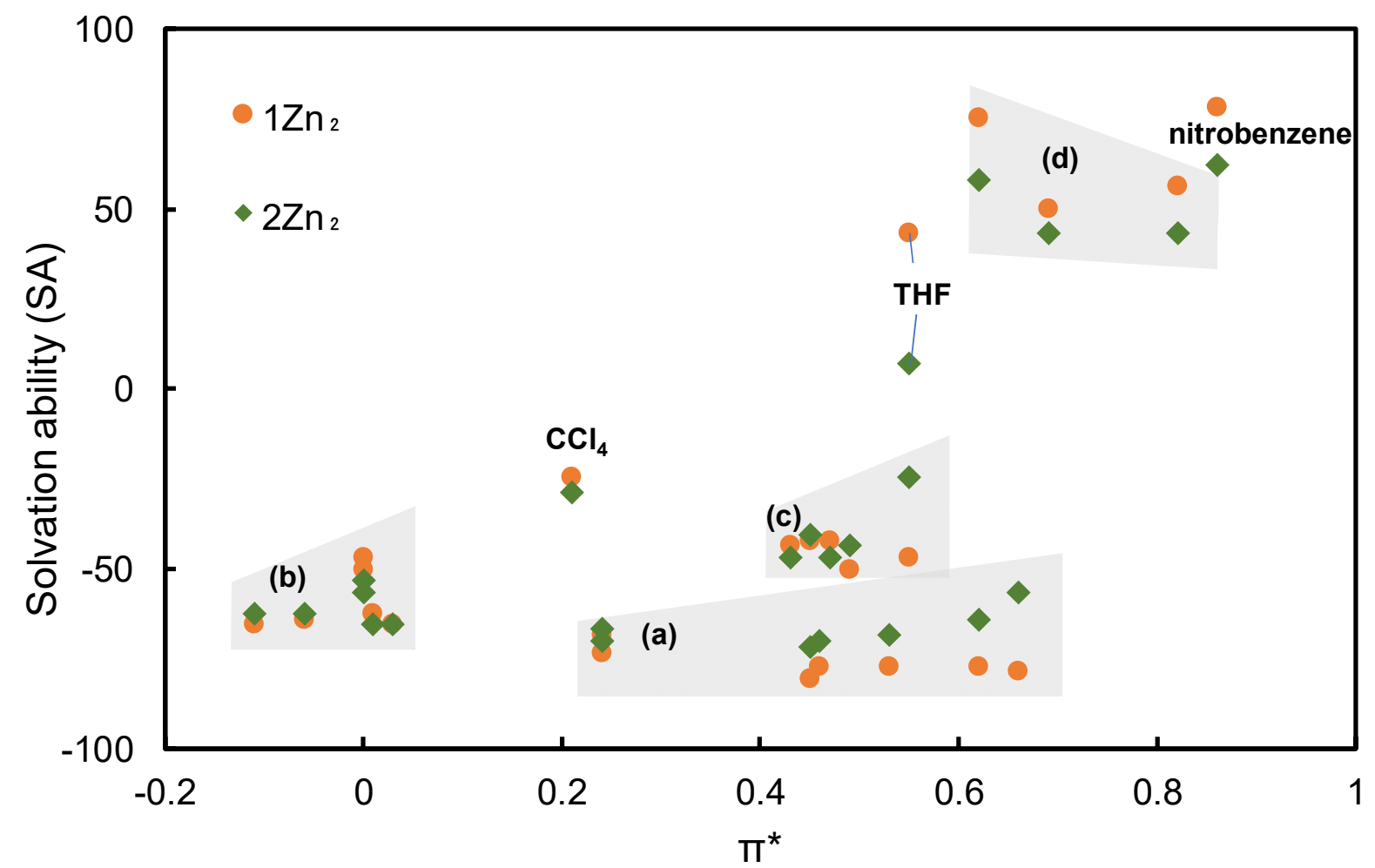

Figure S7. Relationships between SA and Kamlet-Traft solvent parameter $\pi^{*}$ of various solvents in Table S1. (a) polar nonaromatic compounds, (b) nonpolar and nonaromatic compounds (alkanes), (c) benzene and methylated benzenes, (d) chloro-organic compounds. 


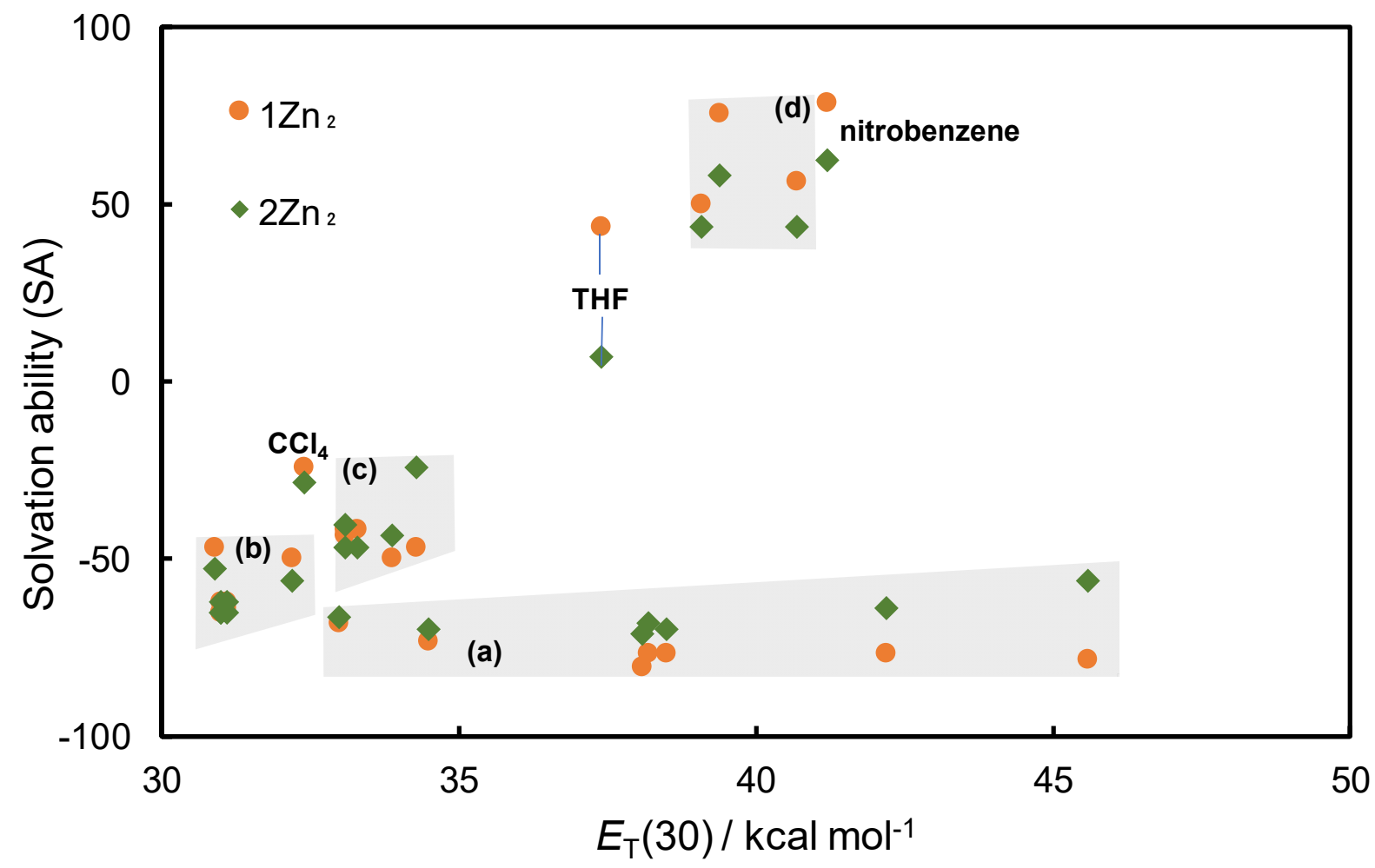

Figure S8. Relationships between SA and solvent polarity parameter $E_{\mathrm{T}}(30)$ of various solvents in Table S1. (a) polar nonaromatic compounds, (b) nonpolar and nonaromatic compounds (alkanes), (c) benzene and methylated benzenes, (d) chloro-organic compounds.

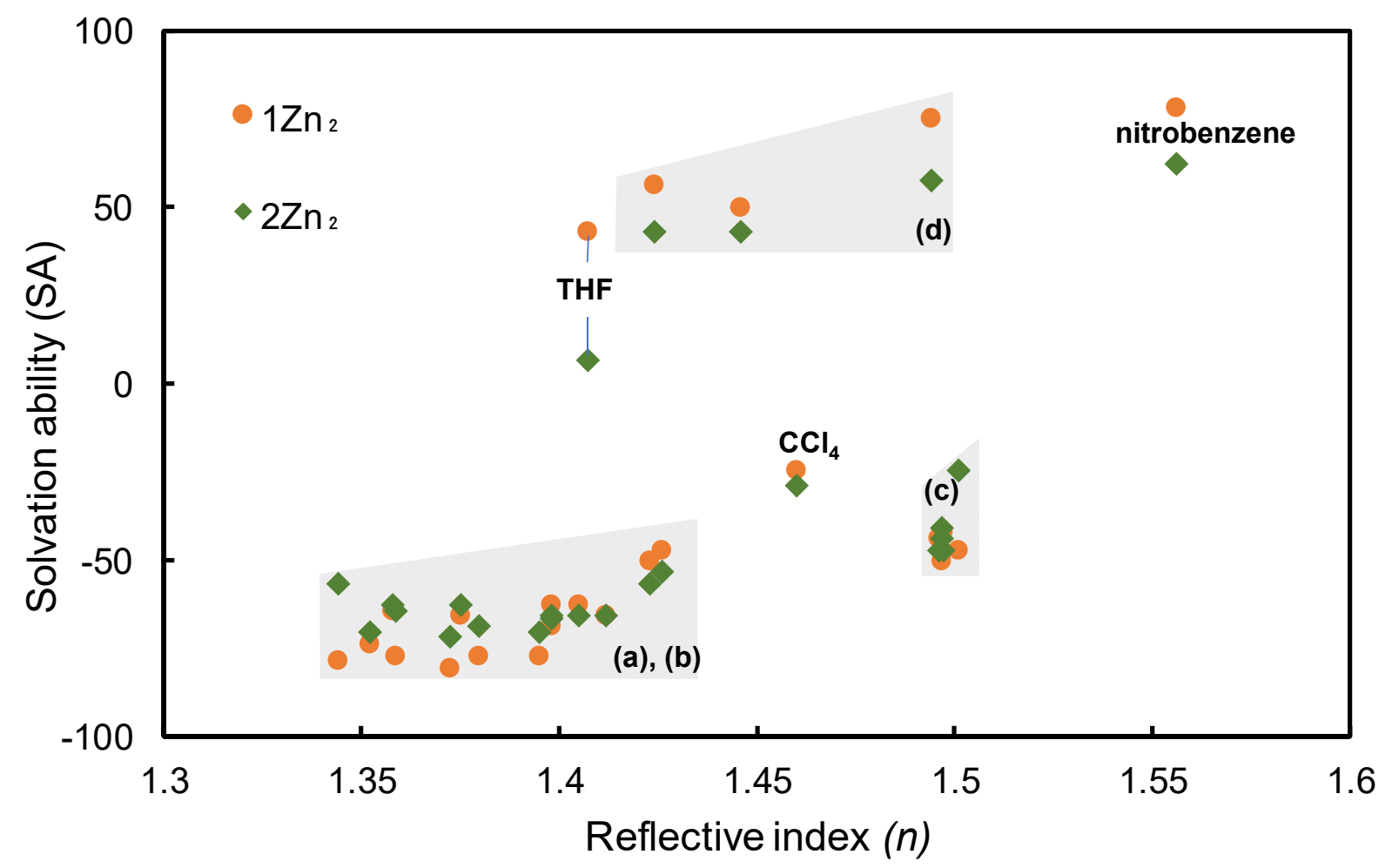

Figure S9. Relationships between SA and reflective index (n) of various solvents in Table S1. (a) polar nonaromatic compounds, (b) nonpolar and nonaromatic compounds (alkanes), (c) benzene and methylated benzenes, (d) chloro-organic compounds. 


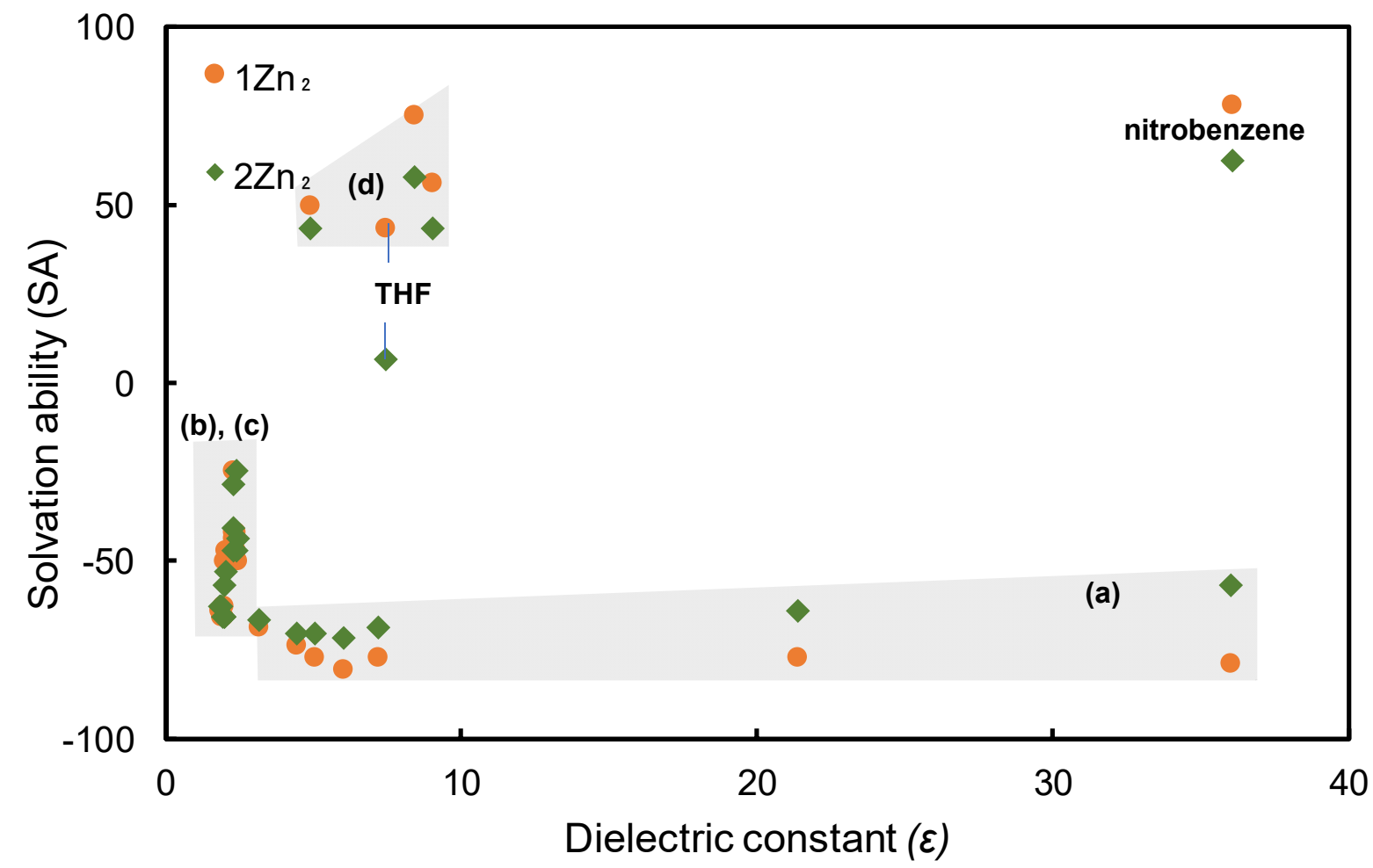

Figure S10. Relationships between SA and dielectric constant $(\varepsilon)$ of various solvents in Table S1. (a) polar nonaromatic compounds, (b) nonpolar and nonaromatic compounds (alkanes), (c) benzene and methylated benzenes, (d) chloro-organic compounds. 


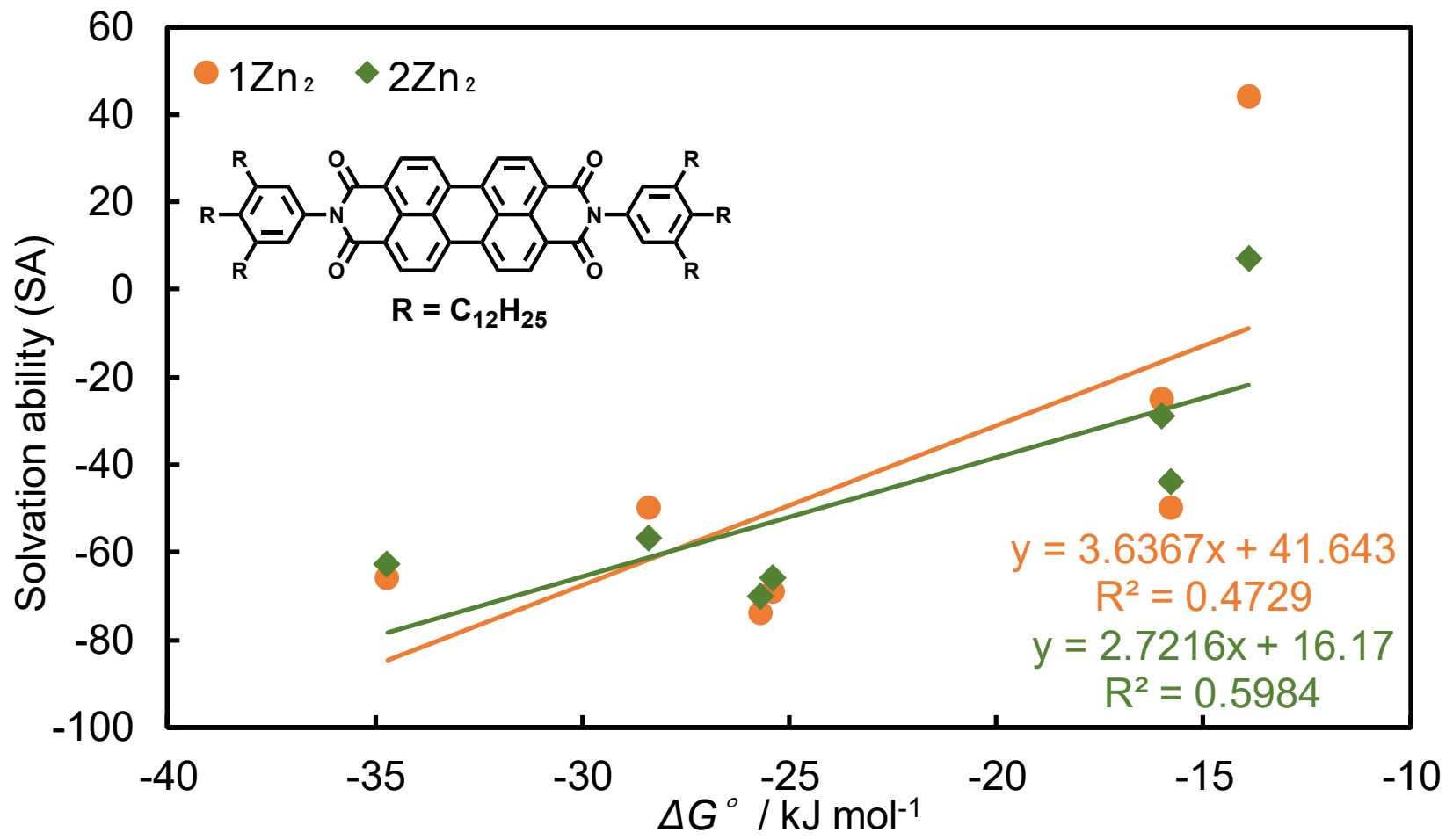

Figure S11. Relationships between SA and $\Delta G^{0}$ for formation of oligomer of perylene bisimide $\mathbf{2}^{2}$.

Table S2. Relationships between SA and $\Delta G^{0}$ for formation of oligomer of perylene bisimide $\mathbf{2}^{2}$.

\begin{tabular}{llll}
\hline & & \multicolumn{2}{c}{ Solvation ability $(\mathrm{SA})$} \\
Solvent & $\Delta G^{\circ} / \mathrm{kJ} \mathrm{mol}^{-1}$ & $1 \mathrm{Zn}_{2}$ & $2 \mathrm{Zn}_{2}$ \\
\hline hexane & -34.7 & -66 & -63 \\
MCH & -28.4 & -50 & -57 \\
dibutyl ether & -25.4 & -69 & -66 \\
diethyl ether & -25.7 & -74 & -70 \\
$\mathrm{CCl}_{4}$ & -16.0 & -25 & -29 \\
toluene & -15.8 & -50 & -44 \\
THF & -13.9 & 44 & 7 \\
\hline
\end{tabular}




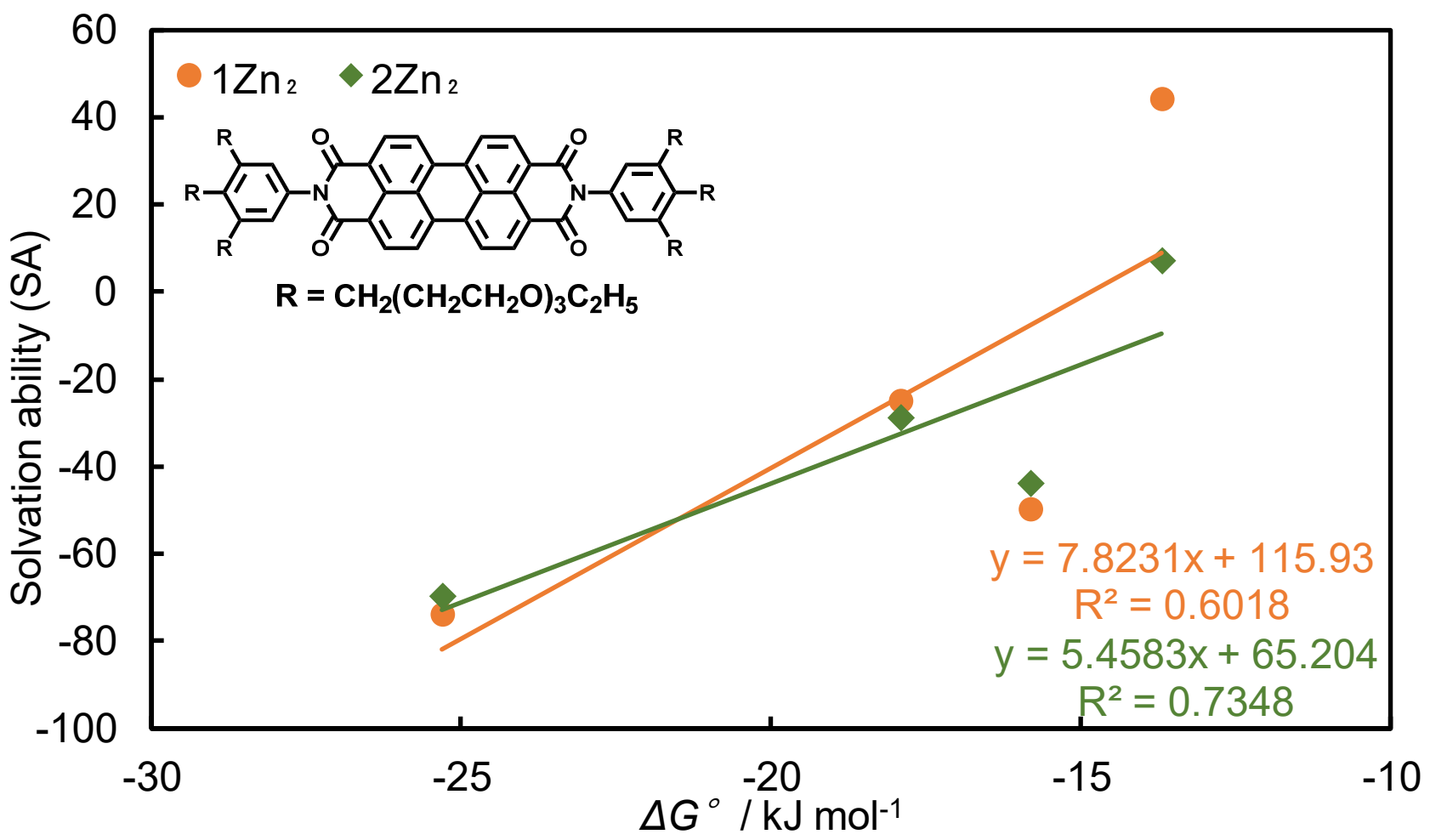

Figure S12. Relationships between SA and $\Delta G^{0}$ for formation of oligomer of perylene bisimide $\mathbf{3}^{2}$.

Table S3. Relationships between SA and $\Delta G^{0}$ for formation of oligomer of perylene bisimide $\mathbf{3}^{2}$.

Solvation ability (SA)

\begin{tabular}{llll} 
Solvent & $\Delta G^{\circ} / \mathrm{kJ} \mathrm{mol}^{-1}$ & $1 \mathrm{Zn}_{2}$ & $2 \mathrm{Zn}_{2}$ \\
\hline diethyl ether & -25.3 & -74 & -70 \\
$\mathrm{CCl}_{4}$ & -17.9 & -25 & -29 \\
toluene & -15.8 & -50 & -44 \\
THF & -13.7 & 44 & 7 \\
\hline
\end{tabular}




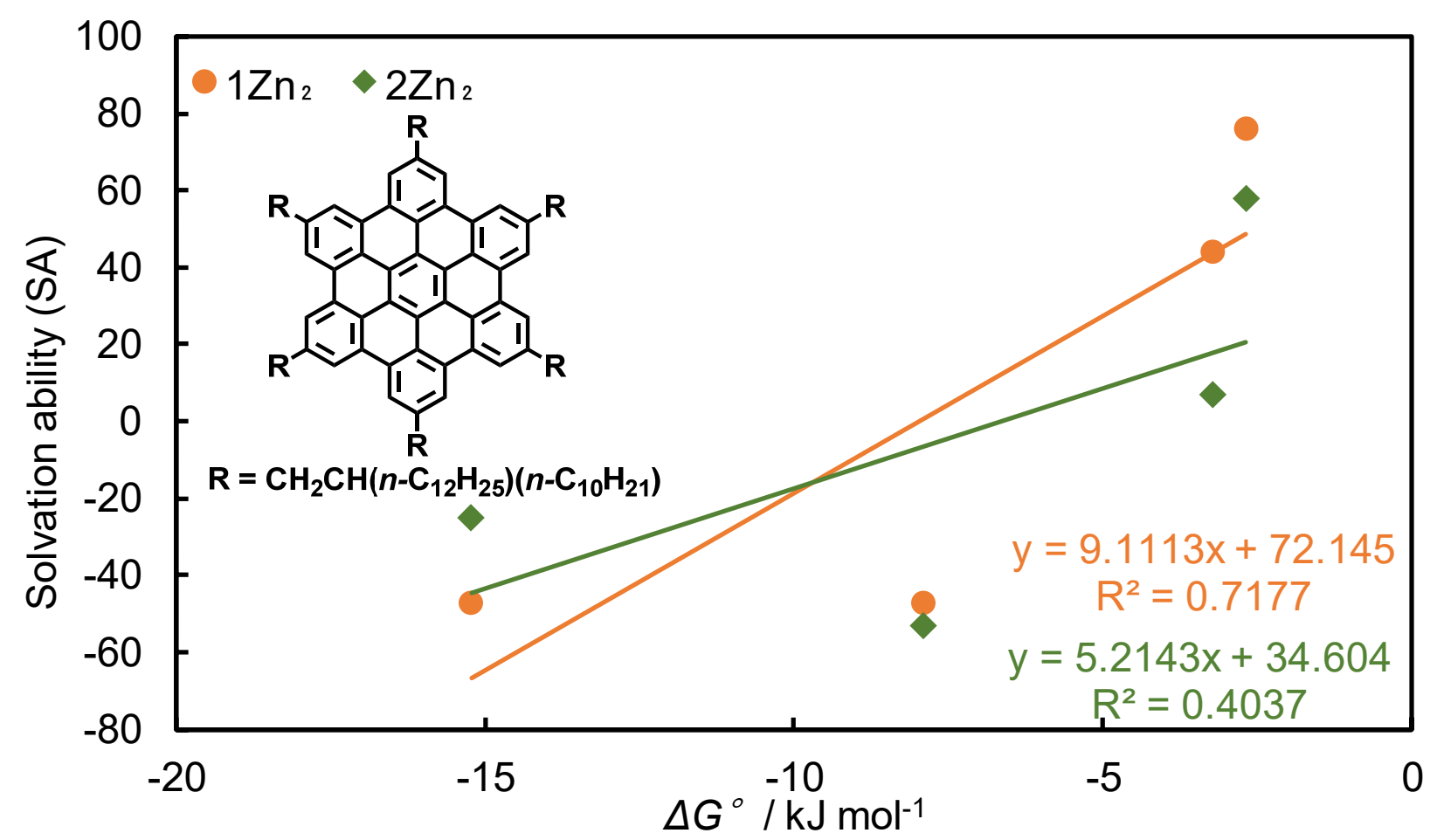

Figure S13. Relationships between SA and $\Delta G^{0}$ for formation of oligomer of hexabenzocoronene $4^{3}$.

Table S4. Relationships between SA and $\Delta G^{0}$ for formation of oligomer of hexabenzocoronene $4^{3}$.

\begin{tabular}{llll}
\hline & & \multicolumn{2}{c}{ Solvation ability (SA) } \\
Solvent & $\Delta G^{\circ} / \mathrm{kJ} \mathrm{mol}^{-1}$ & $1 \mathrm{Zn}_{2}$ & $2 \mathrm{Zn}_{2}$ \\
\hline $1,1,2,2-$ & -2.68 & 76 & 58 \\
teterachloroethane & & & \\
cyclohexane & -7.90 & -47 & -53 \\
THF & -3.23 & 44 & 7 \\
benzene & -15.2 & -47 & -25 \\
\hline
\end{tabular}




\section{References}

1. Abboud, J.-L.; Notario, R.; Commission, I., Critical compilation of scales of solvent parameters. Part I. Pure, non-hydrogen bond donor solvents - Technical report. Pure Appl. Chem. 1999, 71, 645-718.

2. Chen, Z.; Fimmel, B.; Würthner, F., Solvent and substituent effects on aggregation constants of perylene bisimide $\pi$-stacks - a linear free energy relationship analysis. Org. Biomol. Chem. 2012, 10 (30), 5845-5855.

3. Kastler, M.; Pisula, W.; Wasserfallen, D.; Pakula, T.; Müllen, K., Influence of Alkyl Substituents on the Solution- and Surface-Organization of Hexa-peri-hexabenzocoronenes. J. Am. Chem. Soc. 2005, 127 (12), 4286-4296.

4. Lahiri, S.; Thompson, J. L.; Moore, J. S., Solvophobically Driven $\pi$-Stacking of Phenylene Ethynylene Macrocycles and Oligomers. J. Am. Chem. Soc. 2000, 122 (46), 11315-11319. 